\title{
Efficacy of Combined Aromatase Inhibitor and Luteinizing Hormone- Releasing Hormone Agonist in Premenopausal Metastatic Breast
} Cancer

\author{
Sang Hee Kim, Jihye Choi, Chan Sub Park, Hyun-Ah Kim, Woo Chul Noh, Min-Ki Seong \\ Department of Surgery, Korea Cancer Center Hospital, Korea Institute of Radiological and Medical Sciences, Seoul, Korea
}

Purpose: Endocrine therapy is the preferred treatment for hormone receptor (HR)-positive metastatic breast cancer (MBC). We investigated the efficacy of combined aromatase inhibitor (Al) and luteinizing hormone-releasing hormone (LHRH) agonist in premenopausal patients with HR-positive MBC. Methods: We retrospectively analyzed the medical records of $21 \mathrm{HR}$-positive premenopausal MBC patients treated with combined Al and LHRH agonist therapy. Results: The median follow-up period was 32.9 months. The overall response rate was $47.6 \%$, with three complete responses (14.3\%) and seven partial responses (33.3\%). Nine patients (42.9\%) achieved stable disease lasting more than 6 months; thus, the clinical benefit rate was $90.4 \%$. The median time to progression was 45.4 months. No patients experienced grade 3 or 4 toxicity. Conclusion: Combined Al and LHRH agonist treatment safely and effectively induced remission or prolonged disease stabilization, suggesting that this could be a promising treatment option for HR-positive premenopausal patients with MBC.

Key Words: Aromatase inhibitors, Breast neoplasms, Gonadotropin-releasing hormone, Goserelin, Premenopause

\section{INTRODUCTION}

Breast cancer is the most commonly diagnosed malignancy and remains the leading cause of cancer-related deaths among women worldwide. The successful implementation of breast screening programs in many countries has increased the proportion of cancers that are detected at an early stage, which may help to reduce the mortality rate [1]. However, approximately $5 \%-10 \%$ of patients have metastatic breast cancer (MBC) at initial diagnosis, and even women with early-stage breast cancer will eventually develop distant metastasis in $20 \%-30 \%$ of cases $[2,3]$. Unfortunately, MBC is generally regarded as an incurable disease, with a median survival time of only approximately 3 years. Therefore, the primary goal of $\mathrm{MBC}$ treatment is not to achieve a complete cure but to prolong survival, control the disease and its symptoms, and maintain a reasonable quality of life [4-6].

Beatson's [7] seminal discovery in 1896 that some patients with in-

Correspondence: Min-Ki Seong

Department of Surgery, Korea Cancer Center Hospital, Korea Institute of Radiological and Medical Sciences, 75 Nowon-ro, Nowon-gu, Seoul 01812, Korea Tel: +82-2-970-1251, Fax: +82-2-970-2419, E-mail: mklegend@kcch.re.kr

*These authors contributed equally to this work.

This research was supported by grants from the Radiological Translational Research Program (50472-2018).

Received: May 23, 2018 Revised: Jul 10, 2018 Accepted: Aug 12, 2018 operable breast cancer entered into remission following oophorectomy was the foundation for a scientific approach to cancer treatment, and endocrine therapy remains the preferred treatment for hormone-sensitive MBC. Several endocrine therapies have proven to be effective, including use of selective estrogen receptor (ER) modulators, aromatase inhibitors (AIs), pure estrogen antagonists, progestational agents, and ovarian ablation (through surgical oophorectomy, radiation therapy, or luteinizing hormone releasing hormone $[\mathrm{LHRH}]$ analogs) [8]. AI administration is the gold standard treatment for postmenopausal patients with hormone receptor (HR)-positive breast cancer. However, AI is contraindicated for premenopausal women [9]; thus, premenopausal women with HR-positive MBC have relatively few endocrine-based treatment options. According to current guidelines, ovarian ablation or suppression plus endocrine therapy as for postmenopausal women ( \pm cyclin-dependent kinase $4 / 6$ or mammalian target of rapamycin inhibitor), or selective estrogen receptor modulators, are initially recommended in premenopausal patients with MBC [10]. Although AI alone is not indicated for premenopausal women, its use may be possible in premenopausal women who have been rendered postmenopausal by ovarian ablation. The combined results of the TEXT and SOFT trials demonstrated that, compared with tamoxifen, an AI (exemestane) significantly reduced the risk of 
recurrence when used as an adjuvant treatment after the suppression of ovarian function. Consequently, there is a growing debate over the role of $\mathrm{AI}$ in the treatment of premenopausal patients with $\mathrm{MBC}$ [11], and to date, only a few studies have addressed this important issue [1218]. Here, we report the clinical outcomes following combined AI and LHRH agonist treatment for premenopausal patients with MBC.

\section{METHODS}

\section{Patients}

We searched the prospectively maintained, web-based breast cancer databases of Korea Cancer Center Hospital, Republic of Korea, which include information relating to all patients with breast cancer treated since 1983. The eligibility criteria for this retrospective analysis were as follows: (1) premenopausal women who had had regular menses over the preceding 12 months and serum follicle-stimulating hormone (FSH) values consistent with a premenopausal state (serum FSH $<30$ IU/L); (2) a diagnosis of metastatic or recurrent breast cancer; (3) histologically confirmed ER-positive and/or progesterone receptor (PR)-positive disease; (4) treatment with AI plus LHRH agonist for advanced breast cancer; and (5) assessment was possible using the Response Evaluation Criteria in Solid Tumors (RECIST) guideline, version 1.1. A total of 21 eligible patients were identified on the basis of these inclusion criteria between 2008 and 2014. Baseline staging was available for all of these patients, and the treatment response of each patient was evaluated every 3 months. This study was reviewed and approved by the Korea Cancer Center Hospital Institutional Review Board (K-1506-002-023). The need for informed consent was waived owing to the retrospective nature of this study.

Immunohistochemical analysis of ER, PR, and human epidermal growth factor receptor 2 expression

Expression of ER, PR, and human epidermal growth factor receptor 2 (HER2) was evaluated using immunohistochemistry (IHC) immediately after biopsy or surgery. Positive staining for ER or PR was defined as the presence of at least $10 \%$ positively stained nuclei in 10 high-power fields, and HER2 positivity was defined as either an IHC score of $3+$ or HER2 gene amplification detected by fluorescence in situ hybridization. For subgroup analysis according to ER status, the patients were divided into high- or low-ER expression groups. High
ER status was defined as tumor ER expression with an Allred score greater than 7. If the Allred score was unknown, an IHC ER 3+ score was considered to represent a high-ER tumor.

\section{Treatment and adverse events}

The only LHRH agonist used in this study was goserelin (Zoladex; AstraZeneca plc, Cambridge, UK). Goserelin (3.6 mg) was injected subcutaneously in the anterior abdominal wall every 4 weeks. AI including letrozole (either Femara, Novatis, Basel, Switzerland, $2.5 \mathrm{mg}$ or Bretra, Shin Poong Pharmacetual, Seoul, Korea, $2.5 \mathrm{mg}$ ), anastrozole (Arimidex; AstraZeneca plc, $1 \mathrm{mg}$ ), or exemestane (Aromasin; Pfizer, New York, USA, $25 \mathrm{mg}$ ) were administered orally once daily. Adverse events were evaluated according to the Common Terminology Criteria for Adverse Events, version 4.0.

\section{Response evaluation}

This combination therapy was continued until disease progression or intolerable toxicity. Response to treatment was assessed according to the RECIST criteria, version 1.1. Although bone lesions are not usually considered target lesions, in this study, they were treated as target lesions for the evaluation of response in patients with bone-only metastasis. The overall response rate (ORR) was defined as the proportion of patients with a partial response or complete response. The clinical benefit rate (CBR) was defined as the percentage of patients with a complete response, partial response, or stable disease that lasted for at least 6 months.

\section{Statistical analysis}

The chi-square test and Fisher exact test were used to compare categorical variables. Progression-free survival (PFS) was defined as the time from the initial administration of AI plus LHRH agonist until the first documented disease progression or death from any cause. Overall survival (OS) was defined as the interval from the first day of treatment until death from any cause or the last date of follow-up. For survival analyses, the Kaplan-Meier method and log-rank test were used. Multivariate analysis was performed using a Cox proportional hazards regression model. In all tests, a $p$-value $<0.05$ was considered to be statistically significant. 


\section{RESULTS}

\section{Patient characteristics}

A total of 21 premenopausal women with HR-positive MBC were included in this analysis. Patient and tumor characteristics are presented in Table 1. The median patient age at the start of AI plus LHRH agonist treatment was 41 years (range, 29-47 years). Sixteen patients (76.2\%) had an ER-positive/PR-positive tumor and five patients

Table 1. Clinicopathological characteristics of 21 patients

\begin{tabular}{|c|c|}
\hline Characteristic & No. $(\%)$ \\
\hline \multicolumn{2}{|l|}{ Age (at the time of treatment, yr) } \\
\hline$<40$ & $9(42.9)$ \\
\hline$\geq 40$ & $12(57.1)$ \\
\hline \multicolumn{2}{|l|}{ Disease status } \\
\hline Metastatic breast cancer at initial diagnosis & $4(19.0)$ \\
\hline Recurrent breast cancer & $17(81.0)$ \\
\hline \multicolumn{2}{|l|}{ Hormone receptor status } \\
\hline $\mathrm{ER}+$ and $\mathrm{PR}+$ & $16(76.2)$ \\
\hline $\mathrm{ER}+$ and PR- & $5(23.8)$ \\
\hline $\mathrm{ER}-$ and $\mathrm{PR}+$ & 0 \\
\hline \multicolumn{2}{|l|}{ ER status } \\
\hline High & $11(52.4)$ \\
\hline Low & $10(47.6)$ \\
\hline \multicolumn{2}{|l|}{ HER2 status } \\
\hline Positive & $2(9.5)$ \\
\hline Negative & $19(90.4)$ \\
\hline \multicolumn{2}{|l|}{ Prior tamoxifen plus LHRH agonist treatment } \\
\hline Yes & $7(33.3)$ \\
\hline No & $14(66.7)$ \\
\hline \multicolumn{2}{|l|}{ Site of involved disease } \\
\hline Breast & $2(9.5)$ \\
\hline Lymph nodes & $5(23.8)$ \\
\hline Bone & $11(52.4)$ \\
\hline Lung & $9(42.9)$ \\
\hline Pleura & $1(4.8)$ \\
\hline Liver & $5(23.8)$ \\
\hline Bone only & $6(28.6)$ \\
\hline \multicolumn{2}{|l|}{ No. of involved disease sites } \\
\hline 1 & $14(66.7)$ \\
\hline 2 & $4(19.0)$ \\
\hline$\geq 3$ & $3(14.3)$ \\
\hline \multicolumn{2}{|l|}{ Location of involved disease sites } \\
\hline Visceral & $9(42.9)$ \\
\hline Nonvisceral & $12(57.1)$ \\
\hline \multicolumn{2}{|l|}{ Aromatase inhibitor } \\
\hline Letrozole & $19(90.5)$ \\
\hline Anastrozole & $1(4.8)$ \\
\hline Exemestane & $1(4.8)$ \\
\hline
\end{tabular}

$E R=$ estrogen receptor; $\mathrm{PR}=$ progesterone receptor; $\mathrm{HER} 2=$ human epidermal growth factor receptor 2; LHRH= luteinizing hormone-releasing hormone.
(23.8\%) had an ER-positive/PR-negative tumor. According to the criteria described above, approximately half of the patients had a highER tumor (11, 52.4\%). Most patients (19, 90.5\%) had an HER2-negative tumor. Two-thirds of patients $(14,66.7 \%)$ had a single metastasis, more than half had non-visceral disease (12, 57.1\%), and six (28.6\%) had only bony metastasis. Of the 21 patients, seven (33.3\%) had received prior tamoxifen plus LHRH agonist treatment. Among these seven patients, six were treated with letrozole plus LHRH agonist when tamoxifen plus LHRH failed. In one patient, six cycles of doxorubicin and docetaxel were administered due to development of multiple liver metastases. In this patient, letrozole plus LHRH agonist was considered as a next-line regimen following chemotherapy. The AI administered in this study were letrozole (19 patients, 90.5\%), anastrozole (1 patient, 4.8\%), and exemestane (1 patient, 4.8\%). For letrozole, Femara was administered to seven patients and Bretra to 12 patients.

\section{Efficacy}

After the follow-up period, 11 patients experienced disease progression (52.4\%) and five patients died owing to their disease (23.8\%). The median follow-up period was 32.9 months (range, 1.3-82.3 months). The median PFS in all 21 patients was 45.4 months (95\% confidence interval [CI], 16.7-74.2 months) (Figure 1). Owing to the relatively short follow-up period, the median OS could not be evaluated in this study. All patients had an evaluable lesion, as described above, including six patients with only bony metastasis. The response data are sum-

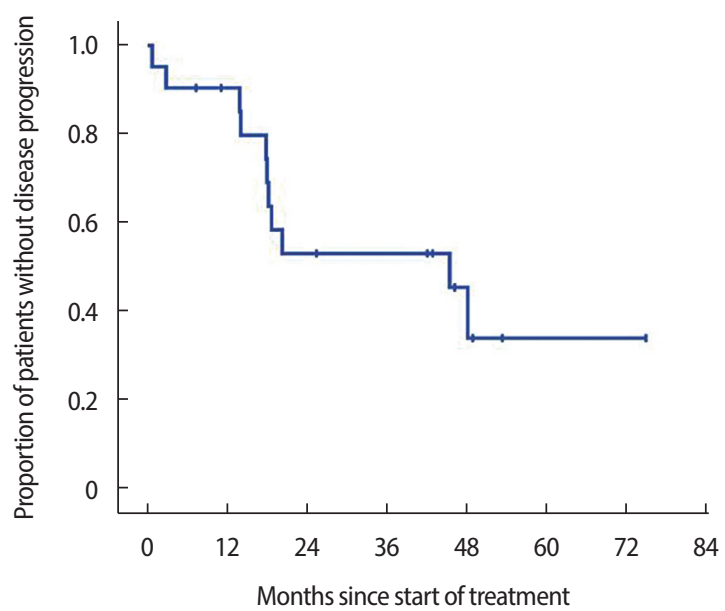

Figure 1. Kaplan-Meier curves of time to progression-free survival. After a median follow-up period of 32.9 months (range, 1.3-82.3 months), 11 patients (52.4\%) experienced disease progression and five patients $(23.8 \%)$ died of their disease. 
Table 2. Treatment efficacy in the 21 patients

\begin{tabular}{lc}
\hline & No. (\%) \\
\hline Treatment response & \\
Complete response & $3(14.3)$ \\
Partial response & $7(33.3)$ \\
Stable disease & $9(42.9)$ \\
Progressive disease & $2(9.5)$ \\
Overall response rate & $10(47.6)$ \\
Clinical benefit rate & $19(90.5)$ \\
\hline
\end{tabular}

marized in Table 2. Of 21 patients, three patients achieved a complete response (14.3\%) and seven patients achieved a partial response (33.3\%). Stable disease for more than 6 months was achieved in nine patients (42.9\%), and disease progression during treatment with AI plus LHRH agonist was seen in two patients (9.5\%). Thus, the ORR and CBR were $47.6 \%$ and $90.4 \%$, respectively. In univariate analysis of factors that influence PFS, including ER status, history of prior tamoxifen plus LHRH agonist treatment, and site of metastatic disease, we did not find any other factors that were associated with treatment outcome.

\section{DISCUSSION}

Our results provide supportive evidence for the use of combined AI and LHRH agonist treatment for premenopausal patients with MBC. For the 21 patients included in our study, the ORR was $47.6 \%$, the CBR (a measure of disease control) was 90.4\%, and the median PFS was 45.4 months. Most adverse events were mild and the treatment was well tolerated. None of our patients experienced a serious adverse event requiring treatment discontinuation. Combined LHRH agonist and AI treatment resulted in outcomes at least as good as those after conventional chemotherapy and provided better quality of life.

In premenopausal patients with $\mathrm{MBC}$, ovarian ablation has been the most common endocrine treatment for over 100 years. Pharmacologically induced ovarian suppression, such as LHRH agonist administration, is considered a medical alternative to oophorectomy [7]. Although the benefits of ovarian suppression alone in premenopausal patients with breast cancer is still under investigation, the benefit of combined tamoxifen and LHRH agonist treatment was demonstrated in a meta-analysis of four randomized trials comparing this treatment to LHRH agonist administration alone [19]. Moreover, a few studies have shown promising results for AI in combination with
Table 3. Summary of previous studies on the treatment efficacy of Al plus LHRH agonist therapy

\begin{tabular}{lclc}
\hline Study & No. of patients & $\begin{array}{c}\text { CBR } \\
(\%)\end{array}$ & $\begin{array}{c}\text { Median TTP/PFS } \\
(\mathrm{mo})\end{array}$ \\
\hline Forward et al. [12] & 14 & 75 & 17 \\
Cheung et al. [13] & 36 & 67 & 12 \\
Carlson et al. [14] & 32 & 71.9 & 8.3 \\
Yao et al. [15] & 52 & 71.1 & 10 \\
Nishimura et al. [16] & 37 & 62.2 & 7.3 \\
Tanaka et al. [17] & 14 & 71.4 & 11 \\
Park et al. [18] & 35 & 77.1 & 9.5 \\
\hline
\end{tabular}

$\mathrm{Al}=$ aromatase inhibitor; $\mathrm{LHRH}=$ luteinizing hormone-releasing hormone; $\mathrm{CBR}=$ clinical benefit rate; $\mathrm{TTP}=$ time to progression; $\mathrm{PFS}=$ progression - free survival.

LHRH agonist [12-18], despite concerns that the use of AI alone in premenopausal women could cause ovarian stimulation [9]. In a prospective phase II trial of 32 premenopausal patients with MBC treated with goserelin plus anastrozole, one participant (3.1\%) experienced a complete response, 11 (34.4\%) experienced partial response, and 11 (34.4\%) experienced stable disease for at least 6 months, achieving a CBR of $71.9 \%$. The median time to progression (TTP) was 8.3 months with no serious adverse events [14]. In another small phase II study, goserelin plus letrozole had efficacy and safety that were comparable to letrozole alone (median TTP, 9.5 months vs. 8.9 months, respectively) in postmenopausal patients with $\mathrm{MBC}$, suggesting that combined AI plus LHRH agonist treatment may be as effective in premenopausal women as it is in postmenopausal women [18]. Previously reported clinical outcomes of patients treated with combined AI plus LHRH agonist therapy are summarized in Table 3.

Compared with previous studies, in this small retrospective study, we observed remarkably longer PFS durations. This may be a result of the relatively favorable clinical characteristics of the patients in this study, who tended to have fewer metastatic sites, good performance status, and biological markers, such as high-ER or HER-2-negative metastasis, that predict a good response to endocrine treatment. It is generally accepted that the degree of ER positivity is directly related to endocrine therapy response [20]. Thus, we hypothesized that patients with high-ER disease would survive longer, on average, although we found no significant difference in survival between patients with high-ER and low-ER disease. Regarding the efficacy of LHRH agonist according to patient age, the International Breast Cancer Study Group Trial VIII and the 2005 Early Breast Cancer Trialists' Collaborative 
Group Oxford Overview [2] demonstrated that the use of LHRH agonist was more beneficial in patients aged 40 years or younger. However, as with ER status, we also found no significant difference in PFS between patients aged $>40$ years and those aged $\leq 40$ years. In addition, none of the other clinicopathological variables that might influence survival, such as the location of metastasis, number of metastatic sites, or HER2 status, were associated with treatment outcome.

For most of the patients in our study (15/21, 71.4\%), AI plus LHRH agonist was administered as a first-line therapy following the diagnosis of recurrence. Only seven patients had been previously treated with tamoxifen plus LHRH agonist as adjuvant or palliative therapy. When stratified by previous exposure to tamoxifen plus LHRH agonist, there was no significant difference in survival outcome. These results may be due to the small sample size of our study and warrant further investigation with a larger patient cohort. Letrozole was the AI most frequently administered in our patients $(19 / 21,90.4 \%)$, owing to its relatively low cost, as combined AI and LHRH agonist therapy is not yet covered by national health insurance in Korea. With regard to ovarian function, although we did not routinely assess ovarian function markers such as estradiol, luteinizing hormone, and FSH, none of our patients had reinitiation of menstruation. Although the assessment of toxicity was limited by the retrospective nature of this study, most recorded adverse events were mild, including hot flashes related to hormone deprivation and multiple arthralgia induced by AI. No cases of grade $3 / 4$ toxicity were observed. Previous studies have also reported that AI combined with LHRH agonist is a safe and well-tolerated treatment, with no serious adverse events [12-18].

Our study has several limitations. First, we performed a retrospective analysis using data from a single institution, which made it difficult to accurately assess ovarian function, symptoms, and toxicity profiles. Second, our study is limited by its small sample size, which may mean that it was statistically too underpowered to permit a meaningful comparison of survival between subgroups according to clinically important baseline characteristics. However, this is one of only a few recent studies that have provided evidence for use of combined AI and LHRH agonist administration as a viable treatment option in premenopausal patients with MBC. Future prospective clinical trials will help to better define the role of this combination regimen.

In conclusion, our results suggest that combination therapy with AI and LHRH agonist is a safe and very effective treatment for premeno- pausal women with HR-positive MBC. Furthermore, in the context of palliative care, this combination therapy could significantly delay disease progression and the need for chemotherapy, leading to prolonged patient survival and a better quality of life.

\section{CONFLICT OF INTEREST}

The authors declare that they have no competing interests.

\section{REFERENCES}

1. Torre LA, Bray F, Siegel RL, Ferlay J, Lortet-Tieulent J, Jemal A. Global cancer statistics, 2012. CA Cancer J Clin 2015;65:87-108.

2. Early Breast Cancer Trialists' Collaborative Group (EBCTCG). Effects of chemotherapy and hormonal therapy for early breast cancer on recurrence and 15-year survival: an overview of the randomised trials. Lancet 2005;365:1687-717.

3. Gennari A, Conte P, Rosso R, Orlandini C, Bruzzi P. Survival of metastatic breast carcinoma patients over a 20 -year period: a retrospective analysis based on individual patient data from six consecutive studies. Cancer 2005; 104:1742-50.

4. Stockler M, Wilcken NR, Ghersi D, Simes RJ. Systematic reviews of chemotherapy and endocrine therapy in metastatic breast cancer. Cancer Treat Rev 2000;26:151-68.

5. Osoba D. Health-related quality of life as a treatment endpoint in metastatic breast cancer. Can J Oncol 1995;5 Suppl 1:47-53.

6. Geels P, Eisenhauer E, Bezjak A, Zee B, Day A. Palliative effect of chemotherapy: objective tumor response is associated with symptom improvement in patients with metastatic breast cancer. J Clin Oncol 2000;18:2395-405.

7. Beatson GT. On the treatment of inoperable cases of carcinoma of the mamma: suggestions for a new method of treatment, with illustrative cases. Trans Med Chir Soc Edinb 1896;15:153-79.

8. Gradishar WJ, Anderson BO, Blair SL, Burstein HJ, Cyr A, Elias AD, et al. Breast cancer version 3.2014. J Natl Compr Canc Netw 2014;12:542-90.

9. Dowsett M, Folkerd E, Doody D, Haynes B. The biology of steroid hormones and endocrine treatment of breast cancer. Breast 2005; 14:452-7.

10. Giordano SH, Elias AD, Gradishar WJ. NCCN guidelines updates: 
breast cancer. J Natl Compr Canc Netw 2018;16(5S):605-10.

11. Pagani O, Regan MM, Walley BA, Fleming GF, Colleoni M, Láng I, et al. Adjuvant exemestane with ovarian suppression in premenopausal breast cancer. N Engl J Med 2014;371:107-18.

12. Forward DP, Cheung KL, Jackson L, Robertson JF. Clinical and endocrine data for goserelin plus anastrozole as second-line endocrine therapy for premenopausal advanced breast cancer. Br J Cancer 2004;90:590-4

13. Cheung KL, Agrawal A, Folkerd E, Dowsett M, Robertson JF, Winterbottom L. Suppression of ovarian function in combination with an aromatase inhibitor as treatment for advanced breast cancer in pre-menopausal women. Eur J Cancer 2010;46:2936-42.

14. Carlson RW, Theriault R, Schurman CM, Rivera E, Chung CT, Phan SC, et al. Phase II trial of anastrozole plus goserelin in the treatment of hormone receptor-positive, metastatic carcinoma of the breast in premenopausal women. J Clin Oncol 2010;28:391721.

15. Yao S, Xu B, Li Q, Zhang P, Yuan P, Wang J, et al. Goserelin plus letrozole as first- or second-line hormonal treatment in premenopausal patients with advanced breast cancer. Endocr J 2011;58:509-16.
16. Nishimura R, Anan K, Yamamoto Y, Higaki K, Tanaka M, Shibuta K, et al. Efficacy of goserelin plus anastrozole in premenopausal women with advanced or recurrent breast cancer refractory to an LHRH analogue with tamoxifen: results of the JMTO BC08-01 phase II trial. Oncol Rep 2013;29:1707-13.

17. Tanaka K, Tokunaga E, Yamashita N, Taketani K, Akiyoshi S, Morita $\mathrm{M}$, et al. Luteinizing hormone-releasing hormone agonist plus an aromatase inhibitor as second-line endocrine therapy in premenopausal females with hormone receptor-positive metastatic breast cancer. Surg Today 2014;44:1678-84.

18. Park IH, Ro J, Lee KS, Kim EA, Kwon Y, Nam BH, et al. Phase II parallel group study showing comparable efficacy between premenopausal metastatic breast cancer patients treated with letrozole plus goserelin and postmenopausal patients treated with letrozole alone as first-line hormone therapy. J Clin Oncol 2010;28:2705-11.

19. Michaud LB, Jones KL, Buzdar AU. Combination endocrine therapy in the management of breast cancer. Oncologist 2001;6:538-46.

20. Ma CX, Sanchez CG, Ellis MJ. Predicting endocrine therapy responsiveness in breast cancer. Oncology (Williston Park) 2009;23: $133-42$. 